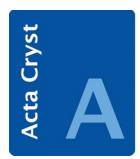

FOUNDATIONS

ADVANCES

ISSN 2053-2733

\title{
Bounding the regularity radius for regular crystals
}

\author{
Dirk Frettloeh*
}

Technische Fakultät, Universität Bielefeld, Germany. *Correspondence e-mail: frettloe@math.uni-bielefeld.de

Received 8 November 2018

Accepted 9 November 2018

Keywords: Delone sets; regularity radius; crystallinity; Engel sets.

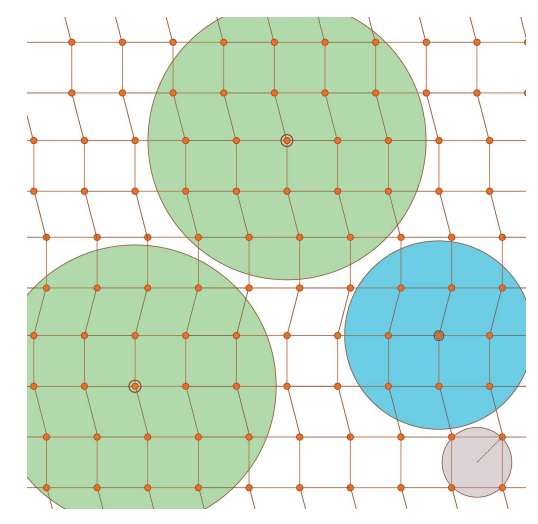

(C) 2019 International Union of Crystallography
A central question in crystallography is how (or if) a globally crystallographic pattern - a crystal - can be determined by local rules. Therefore it is natural to ask 'how far is local?'. In the previous issue of Acta Cryst. A, Baburin et al. (2018) give a partial answer for a particular class of (mathematical, idealized) crystals: they consider only 'regular systems'. A regular system is essentially a crystal with only one type of atom, where each atom is surrounded in the same way by its neighbours. They show that in arbitrary dimension $d$ 'local' means at least $2 d R$. Here $R$ denotes the radius of the largest empty ball in the crystal (compare the grey ball in Fig. 1). For instance, for the primitive lattice $\mathbb{Z}^{2}$ in dimension two we obtain $d=2$ and $R=2^{1 / 2} / 2$, and hence $2 d R=2\left(2^{1 / 2}\right)$. The result says for this example that we need to know at least all neighbourhoods of each atom with radius $2\left(2^{1 / 2}\right)$ in order to ensure that it is indeed a crystal.

Commonly used mathematical models for crystals (or quasicrystals, or more general structures) are Delone sets. A Delone set is an (infinite, discrete) point set $X$ such that (i) there is $r>0$ such that each open ball of radius $r$ contains at most one point of $X$, and (ii) there is $R>r>0$ such that each closed ball of radius $R$ contains at least one point of $X$. The points of $X$ represent the (ideal) atomic positions of some structure. One milestone in mathematical crystallography is the Local Theorem (Delone et al., 1976): it provides a necessary and sufficient local condition for a Delone set $X$ being a crystal. In a nutshell this result states that the Delone set $X$ is a crystal if and only if the number of different local patterns of $X$ of radius $\rho$ stays bounded if $\rho$ tends to infinity. For a more precise version of the statement see Delone et al. (1976).

In order to count the number of different local patterns properly it is useful to define the cluster-counting function. For $x \in X$ let $C(\rho, x)$ denote the (centred) $\rho$-cluster $X \cap B(\rho, x)$, where $B(\rho, x)$ denotes the ball of radius $\rho$ centred in $x$. The cluster-counting function $N(\rho, X)$ is the number of different (centred) $\rho$-clusters in $X$. Note that it is important to consider centred clusters: for instance, in the integer lattice $\mathbb{Z}^{2}$ there are several different $\rho$-clusters of the form $\mathbb{Z}^{2} \cap B(\rho, x)$ if $x$ is arbitrary, but there is only one kind of $\rho$-cluster $C(\rho, x)$ for any particular value $\rho$ if $x$ is required to lie in $\mathbb{Z}^{2}$.

From now on we consider this particular case where there is only one kind of $\rho$-cluster. If $\rho$ is very small $(e . g . \rho \leq r)$ this does not imply anything on $X$ : all $\rho$-clusters in $X$ consist of only one point. If $\rho$ is large, and there is only one kind of $\rho$-cluster in $X$ (up to congruence), then by the Local Theorem $X$ is necessarily crystallographic. Hence it is natural to ask for good (upper and lower) bounds on the value $\widehat{\rho_{d}}$ such that, if there is only one $\rho$-cluster in some Delone set $X$ in $d$-dimensional Euclidean space, then $X$ is necessarily crystallographic. Thus $\widehat{\rho_{d}}$ depends on the dimension $d$, but is universal for all Delone sets $X$ in $d$-dimensional Euclidean space.

A commonly used mathematical model of a crystal is the orbit of one point, or of several (inequivalent) points under a crystallographic group in $d$-dimensional Euclidean space. In the first case the corresponding (infinite) Delone set is called a regular system, in the latter case the Delone set is called a multiregular system. One particular instance of the question of the origin of crystallinity is to find good bounds on the value $\widehat{\rho_{d}}$ described above for regular systems. Let us call the smallest such $\widehat{\rho_{d}}$ the regularity radius (of all regular systems $X$ in a given dimension $d$ ).

Since we may scale any crystallographic Delone set $X$ arbitrarily, the bounds on $\widehat{\rho_{d}}$ ought to be expressed in terms of $R$ (the radius of the largest empty ball in $X$ ). In dimensions $d=1$ and $d=2$ the exact values of the corresponding $\widehat{\rho_{d}}$ are known: $\widehat{\rho_{1}}=2 R$ and $\widehat{\rho_{2}}=4 R$ (see e.g. Barburin et al., 2018; Dolbilin, 2018). Similar arguments as in 


\section{scientific commentaries}

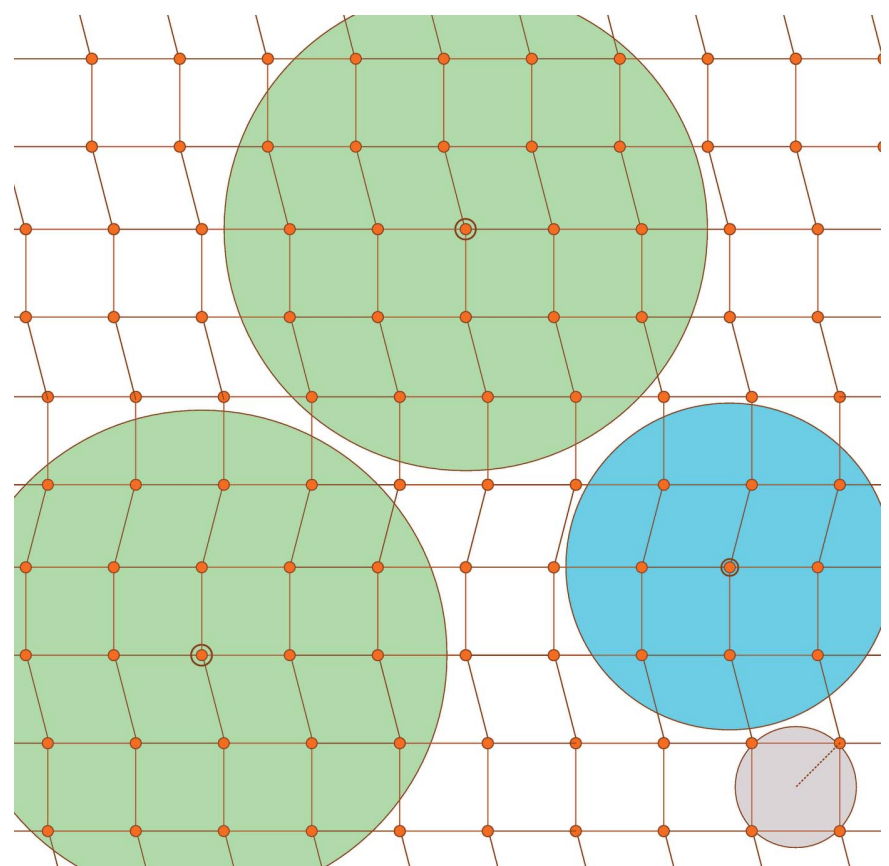

Figure 1

The orange points form a non-crystalline point set. The green balls of radius $2\left(2^{1 / 2}\right)$ can detect the non-crystallinity of the point set. Smaller balls like the blue one cannot: they all look alike.

Dolbilin (2018) yield that $\widehat{\rho}_{d}$ is at least $4 R$ for any $d \geq 2$. Engel conjectured that in dimension three we have $4 R \leq \widehat{\rho}_{3} \leq 6 R$ (Engel, 1986).

A good lower bound on $\widehat{\rho}_{d}$ for Delone sets in arbitrary dimension is obtained in Barburin et al. (2018): it is shown that $\widehat{\rho_{d}}$ is at least $2 d R$ (Theorem 5.8). In particular, $\widehat{\rho_{d}}$ grows at least linearly in the dimension $d$. Hence there is no general bound on $\widehat{\rho_{d}}$ independent of $d$. The result is obtained by a sophisticated construction of Delone sets $X$ ('Engel sets') with only one kind of centred $\rho$-cluster of radius $\rho<2 d R$ such that $X$ still is not a regular system. The construction works in any dimension $d \geq 3$.

This recent result shows that there are still profound questions and answers found in mathematical crystallography today. A next step might be to treat the corresponding question for multiregular systems. Here one cannot expect a regularity radius $\widehat{\rho_{d}}$ such that beyond that radius there exists only one congruence class of $\rho$-clusters. Rather one would require that beyond $\widehat{\rho_{d}}$ there are at most $m$ types of $\rho$-clusters, where $m$ is the number of different orbits with respect to the underlying crystallographic group.

\section{References}

Baburin, I. A., Bouniaev, M., Dolbilin, N., Erokhovets, N. Y., Garber, A., Krivovichev, S. V. \& Schulte, E. (2018). Acta Cryst. A74, 616629.

Delone, B. N., Dolbilin, N. P., Stogrin, M. I. \& Galiulin, R. V. (1976). Sov. Math. Dokl. 17, 319-322.

Dolbilin, N. P. (2018). Discrete Geometry and Symmetry, edited by M. D. E. Conder, A. Deza \& A. Ivic Weiss, Springer Proceedings in Mathematics and Statistics, Vol. 234, pp. 109-125. Springer International Publishing.

Engel, P. (1986). Geometric Crystallography, an Axiomatic Introduction to Crystallography. Dordrecht: Kluwer. 